\title{
Recent advances in radiation oncology
}

\author{
Cristina Garibaldi', Barbara Alicja Jereczek-Fossa ${ }^{2,3}$, Giulia Marvaso ${ }^{2}$, Samantha Dicuonzo ${ }^{2,3}$, Damaris Patricia Rojas ${ }^{2,3}$, Federica $^{2}$ \\ Cattani' ${ }^{1}$, Anna Starzyńska ${ }^{4}$, Delia Ciardo ${ }^{2}$, Alessia Surgo ${ }^{2}$, Maria Cristina Leonardi ${ }^{2}$ and Rosalinda Ricotti ${ }^{2}$
}

'Unit of Medical Physics, European Institute of Oncology, 20141 Milan, Italy

2Department of Radiation Oncology, European Institute of Oncology, 20141 Milan, Italy

${ }^{3}$ Department of Oncology and Hemato-Oncology, University of Milan, 20122 Milan, Italy

${ }^{4}$ Department of Oral Surgery, Medical University of Gdańsk, 80-211 Gdańsk, Poland

Correspondence to: Giulia Marvaso. Email: giulia.marvaso@ieo.it

\begin{abstract}
Radiotherapy (RT) is very much a technology-driven treatment modality in the management of cancer. RT techniques have changed significantly over the past few decades, thanks to improvements in engineering and computing. We aim to highlight the recent developments in radiation oncology, focusing on the technological and biological advances. We will present state-of-the-art treatment techniques, employing photon beams, such as intensity-modulated RT, volumetric-modulated arc therapy, stereotactic body RT and adaptive RT, which make possible a highly tailored dose distribution with maximum normal tissue sparing. We will analyse all the steps involved in the treatment: imaging, delineation of the tumour and organs at risk, treatment planning and finally image-guidance for accurate tumour localisation before and during treatment delivery. Particular attention will be given to the crucial role that imaging plays throughout the entire process. In the case of adaptive RT, the precise identification of target volumes as well as the monitoring of tumour response/modification during the course of treatment is mainly based on multimodality imaging that integrates morphological, functional and metabolic information. Moreover, real-time imaging of the tumour is essential in breathing adaptive techniques to compensate for tumour motion due to respiration.

Brief reference will be made to the recent spread of particle beam therapy, in particular to the use of protons, but also to the yet limited experience of using heavy particles such as carbon ions.

Finally, we will analyse the latest biological advances in tumour targeting. Indeed, the effectiveness of RT has been improved not only by technological developments but also through the integration of radiobiological knowledge to produce more efficient and personalised treatment strategies.
\end{abstract}

Keywords: image guided radiotherapy, adaptive radiotherapy, stereotactic body radiotherapy, intensity-modulated radiotherapy, radiogenomics

Published: 30/11/2017

Received: 02/05/2017

ecancer 2017, 11:785 https://doi.org/10.3332/ecancer.2017.785

Copyright: $\odot$ the authors; licensee ecancermedicalscience. This is an Open Access article distributed under the terms of the Creative Commons Attribution License (http://creativecommons.org/licenses/by/3.0), which permits unrestricted use, distribution, and reproduction in any medium, provided the original work is properly cited. 


\section{Introduction}

Radiotherapy (RT) plays a crucial role in the care of cancer with approximately $50 \%$ of all patients benefiting from RT in the management of their disease [1-3].

Radiation oncologists work with medical and surgical oncologists to coordinate a multidisciplinary approach to the management of cancer.

In the curative setting, RT can be offered as the sole radical treatment. It can also be combined with surgery, being given during (intraoperative), before (neoadjuvant) or after resection (adjuvant), or with systemic therapy, sometimes for organ preservation (such as larynx, breast, urinary bladder, etc) [4-6]. Moreover, it can provide symptom relief in cancers that are locally advanced or disseminated by reducing or eliminating pain from bone metastases in $60 \%$ of cases [7-9].

RT also has an effect on the dissemination of the tumour in that local/regional therapies are, in effect, 'stopping metastases at their source' [10]. More recently, the possibility of the abscopal effect has been raised on the basis of a remission in out-target lesions after localised RT [11].

RT is very much a technology-driven treatment modality. RT techniques have changed significantly over the past few decades due to the improvements in engineering and computing, evolving from conventional irradiation using simple treatment fields towards highly conformal RT techniques, such as intensity-modulated RT (IMRT), intensity-modulated arc therapy (IMAT) and stereotactic RT (SRT), which aim to improve the outcome by escalating the dose to the target and minimizing the toxicity to normal tissue and critical organs. So, nowadays, certain tumours (i.e. breast and prostate cancer) receive shorter courses of RT as a safe and well-tolerated alternative to the longer conventional schemes; this is a big advantage for patients and also for healthcare costs [12]. Indeed, high-precision extremely hypo-fractionated RT has been called virtual surgery, since in many situations it can have a radical curative effect locally that is similar to surgery. From the biological point of view, such a high dose per fraction induces different radiobiological mechanisms of cell killing and therefore introduces a new concept of radio ablation.

Technological advances have mainly been the result of integration of imaging information in every phase of the treatment, from simulation to planning to delivery. Indeed, treatment planning systems (TPS) provide sophisticated image registration and fusion algorithms [13]. Moreover, treatment planning optimisation is becoming more radiobiology-oriented, integrating local radiation damage models [14].

At present, the precise identification of target volumes for treatment planning is mainly based on the integration of radiological/metabolic imaging, such as magnetic resonance imaging (MRI) or positron emission tomography (PET), with computed tomography (CT) scan simulation [15-20]. Tumour localisation immediately before and during treatment delivery by means of image-guided techniques is becoming a part of clinical practice and is a fundamental prerequisite for high-precision RT [21-24]. As part of a comprehensive RT treatment process, adaptive RT (ART) techniques make it possible to modify the treatment plan during the course of RT in order to account for anatomical and biological changes $[13,25]$.

However, technological advances seem likely to reach a plateau in the near future. Indeed, if the tendency towards ever-more cost-effective treatments is to be continued, these must be linked to a better understanding of tumour biology. Although DNA damage is generally considered the primary event leading to radiation-induced cell lethality, numerous non-DNA-related mechanisms have recently been implicated in cellular response to radiation (i.e. the bystander effect and the radiation-induced signalling of epidermal growth factor receptor [26-28].

Many of the developments in understanding the effects of radiation are now leading to a new vision of targeted therapeutics, creating a challenge which we will probably be facing in the near future [29, 30].

In this article, we will review some of the advances that have led to modern high-precision RT as it is today, focusing on the discoveries that have been made in terms of new technology and cancer biology.

\section{Principle of radiotherapy}

In RT, high-energy radiation is used to destroy cancer cells, by depositing energy that damages the genetic material of cells and blocks their ability to divide and proliferate further [31]. 
Photon beams, produced by linear accelerators (LINACs), are characterised by a high deposit of energy near to the body surface with an exponential decrease of energy release as a function of depth. One of the major limitations of photon RT is that the cells of solid tumours become deficient in oxygen. Tumour cells in a hypoxic environment may be as much as 2-3 times more resistant to radiation damage than those in a normal oxygen environment, and much research has been devoted to overcoming hypoxia [32].

In particle therapy, or hadrontherapy, radiation is propagated by travelling corpuscles such as protons or boron, carbon, neon ions [33] that have an antitumour effect independent of tumour oxygen supply because they act mostly via direct energy transfer that causes doublestranded DNA breaks. Compared to photons, charged particles have an inverted depth dose profile; that is, low incident energy deposition with a spike at the tail-end of its dose distribution (the Bragg peak) and essentially no dose beyond the end range. Consequently, hadrontherapy spares the uninvolved tissue distal to the target and generally deposits a lower dose to the tissue proximal to the target than do photons.

The goal of RT is to maximise the radiation dose to cancer cells while minimizing exposure to adjacent normal cells, hence achieving a high probability of local tumour control [tumour control probability (TCP)] with a low risk of normal tissue complications [normal tissue complication probability (NTCP)] [34]. RT is based on the idea that the DNA repair capacity is generally greater in healthy cells than in cancerous cells $[35,36]$.

Cell survival after RT is modelled by an exponential function that accounts for both direct, called alpha, and indirect, called beta, mechanisms of DNA damage. The alpha/beta ratio of these types of damage expresses the ability of the tissue to repair the damage. This repair ability is inversely proportional to the alpha/beta ratio. Historically, the RT schedules conceived exploited the difference in damage repair between tumour and normal tissue by delivering small doses of radiation over a prolonged period of time. The rationale for fractionated RT is based on the four Rs of RT: re-assortment, repair, re-oxygenation and repopulation [37], to which radiosensitivity was later added [38]. Tissue with a high alpha/beta ratio is less sensitive to a high dose per fraction or hypo-fractionated RT compared to tissue with a low alpha/ beta ratio. Most tumours have a high alpha/beta ratio (about $10 \mathrm{~Gy}$ ) and are therefore treated with the standard fractionation of 2 Gy/fraction, or with a hyper-fractionated regimen (dose/fraction $<2 \mathrm{~Gy}$, usually given twice a day). In the past decade, experimental and clinical data have suggested that prostate and breast cancer may actually have a lower alpha/beta ratio than previously suspected [39-43]. This may be due not only to different tumour characteristics but also to cell variability within the tumour or to uncontrolled confounding factors, such as the presence of tumour hypoxia, repopulation or patient-to-patient variability [14].

Recent technological advances have made it possible to safely escalate the dose/fraction, which improves the therapeutic ratio of RT, that is, it increases the cure rate and reduces toxicity $[43,44]$.

\section{Technological advances}

In the following, we will briefly present the high-precision RT techniques currently available in clinical practice together with the fundamental prerequisite to accurately localise the target volume during treatment planning and delivery. Finally, ART is introduced within the framework of personalised medicine.

\section{Treatment techniques: state of the art}

\section{Intensity-modulated radiotherapy}

Intensity modulation [45] was introduced in the early 1990s as a further refinement in the delivery of three-dimensional conformal radiation therapy (3D-CRT). IMRT was made possible by use of computer-controlled multi-leaf collimators (MLCs) and advanced treatment planning optimisation algorithms that are able to create the desired dose variation inside the radiation field. As opposed to standard planning techniques, where the dose distribution can only be modified by means of a try and error approach (changing for instance the field weight, angle and shape), with IMRT, the radiation oncologist designates the doses and dose/volume constraints for the tumour and the surrounding normal organs and the TPS determines the optimal fluence of each field resulting in a tailored dose distribution (inverse planning). 
In the past, IMRT was usually delivered using a conventional LINAC with a static field geometry. Developments in IMRT techniques have focused on reducing treatment times with arc therapy by converting multiple static field IMRT into continuously rotating gantry intensity modulation [46].

Examples of IMAT solutions include TomoTherapy Hi-Art (Accuray, Inc., Sunnyvale, CA, USA) [47], and volumetric-modulated arc therapy (RapidArc, Varian Medical Systems, Inc., Paolo Alto, CA, USA and VMAT, Elekta, AB, Stockholm, Sweden) [48].

IMRT/IMAT techniques make it possible to deliver different levels of dose to different parts of the tumour (for instance, a hypoxic area of the tumour, identified by means of functional imaging, may receive a boost dose). Approaches using a simultaneous integrated boost (SIB) and the delivery of dose-escalated conventionally fractionated or hypo-fractionated RT using IMRT techniques are now being investigated as an alternative to conventional RT for different anatomical sites: breast [49, 50], head and neck [51, 52] prostate [53, 54]. Examples, using tomotherapy, of SIBs delivered to the breast tumour bed and to the dominant intra-prostatic lesions (DILs) are shown in Figures 1 and 2.

However, with IMAT techniques, a larger volume of normal tissue receive a low radiation dose (dose bath) compared to static IMRT with a potential increase in induced second malignancies. Hall and Wuu [55] reported a theoretically increased risk of second malignancy from $1 \%$ to $1.75 \%$. It is important to note that numerous epidemiological studies estimate that a large proportion of second cancers are related to other factors, such as lifestyle (smoking, alcohol, obesity, physical inactivity and diet), infections, (human papilloma virus, hepatitis $\mathrm{C}$ virus, etc) or genetics [56].

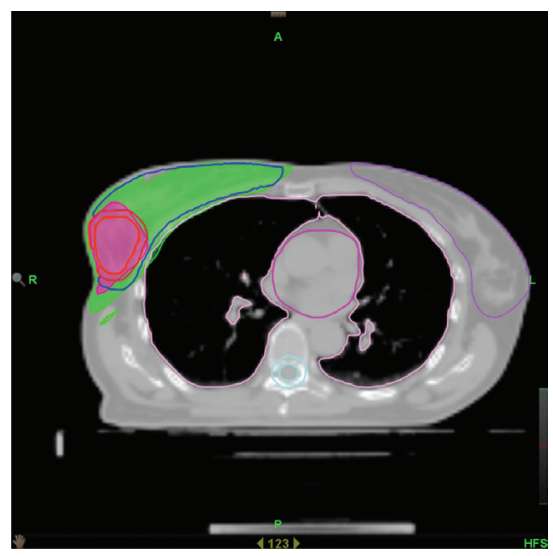

Figure 1. Axial view of a treatment plan for whole breast irradiation with SIB delivered with TomoTherapy. The colour green corresponds to $95 \%$ of the prescribed dose to the breast, and the colour red to $95 \%$ of the boost dose.

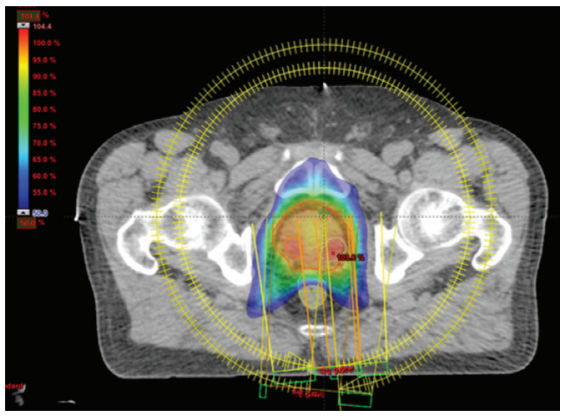

Figure 2. Axial view of a volumetric arc therapy (VMAT) treatment plan for prostate cancer delivered with RapidArc, with SIB to DILs. 


\section{Stereotactic body radiotherapy}

Stereotactic body RT (SBRT) is very much a technology-driven treatment modality [57]. SBRT systems are capable of producing very conformal treatment plans with a steep dose gradient outside the target. This technique makes possible safe and efficacious treatment across a broad array of anatomic locations, in proximity to critical organs, and even adjacent to or within prior RT fields. Essential requirements for SBRT are the accuracy of target delineation (see 'Tumour Localisation in Treatment Planning' section), and the implementation of inter- and intra-fraction tumour motion compensation strategies (especially for tumours in the lung and in the upper abdomen). The wider availability of in-room imaging and advanced treatment delivery systems means that more institutions are now offering SBRT (see 'Tumour Localisation in Treatment Delivery' section) [58]. At present, there are a variety of systems available for SBRT. Some of them are based on the traditional LINAC gantry, for example, Versa HD (Elekta, AB, Stockholm, Sweden) [59] and TrueBeam STx (Varian Medical Systems, Inc., Paolo Alto, CA, USA) [60], whereas others have moved away from this design in search of greater non-coplanar beam arrangements, for example, CyberKnife ${ }^{\circledR}$ (Accuray, Inc., Sunnyvale, CA, USA) [61] and VERO (Mitsubishi Heavy Industries, Ltd., Japan, and BrainLAB AG, Feldkirchen, Germany) $[62,63]$. An example of a stereotactic treatment plan delivered with CyberKnife ${ }^{\circledR}$ for early-stage non-small cell lung carcinoma is shown in Figure 3.

The local ablative capability of SBRT challenges surgery as the gold standard and could become the standard for patients with early stage lung cancer, who are operable but are at high risk of morbidity [64]. Indeed, SBRT has been called virtual surgery or radio ablation as in many cases, such as lung, it can have a radical curative effect locally that is similar to surgery.

\section{Particle beam therapy}

The past decade has seen an increasing use of particle therapy, particularly protons [65]. Radiation dose distributions for proton therapy often appear to be better than those for IMRT photon-based treatments, particularly in that they reduce the low and intermediate radiation dose to normal tissue. Proton therapy is new and although it has dosimetric advantages theoretically, independent evaluation is still to be made in order to assess its strengths and weaknesses [66,67]. Indeed, prospective clinical trials to compare proton therapy to photon IMRT need to be carried out. Moreover, proton therapy is in the midst of a significant technological development at the level of motion management, evolving from passively scattered beams towards actively scanned ones [68-70]. Finally, proton therapy could be of use in a stereotactic regimen, but at the moment there is no clinical series that supports this hypothesis.

Proton therapy has been used internationally for cancers of the eye, base of skull and spine, particularly in paediatric patients [66, 71-73]. Indeed, proton therapy in children has been shown to have a lower incidence of vision and hearing impairment, of neurocognitive degeneration and of second cancers, than is the case with other RT modalities.

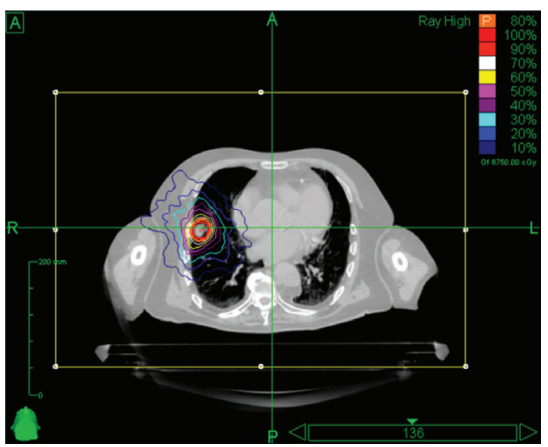

Figure 3. Axial view of a stereotactic treatment plan delivered with CyberKnife for an early stage non-small cell lung carcinoma. 
Moreover, heavy particles, such as carbon ions, are particularly indicated for severely radio-resistant tumours because their biological effectiveness is greater than that of photons and protons [74]. According to the Particle Therapy Co-Operative Group (PTCOG, www.ptcog.com), which constantly updates the statistics on cancer treatment with particle therapy, ten carbon ion therapy facilities are in operation to date (July 2017): five in Japan, two in China and five in Europe (Austria, Germany and Italy). The National Institute of Radiological Sciences Chiba, Japan, has been treating cancer with high-energy carbon ions since 1994, with more than 10,000 patients treated by August 2016 and, thus, is the centre with the greatest experience in carbon ion treatment worldwide $[75,76]$. For the first time, at the National Centre for Oncological Hadrontherapy in Pavia, Italy, carbon ions delivered with active scanning together with breathing synchronisation and rescanning modalities have been used to treat patients with tumours of the liver and pancreas [77].

\section{Advances in treatment planning}

The advent of sophisticated treatment delivery techniques has made it necessary to develop advanced TPSs.

Techniques that can integrate images acquired at different times is becoming a part of common clinical practice for accurate tumour localisation, 4D treatment planning and ART (see 'Tumour Localisation in Treatment Planning' and 'Tumour Localisation in Treatment Delivery' sections). Image registration and fusion, that is, the ability to identify corresponding spatial locations in two or more image volumes and to visualise the result by superimposing the images, is essential in a modern TPS [13]. A crucial aspect, when using registration techniques, is the accuracy of image realignment. Rigid registration algorithms perform translations, rotations and affine image transformations and are widely used in the clinical setting. However, since the human body is intrinsically deformable, rigid techniques often provide insufficient registration accuracy. Thus, elastic or deformable methods are required to cope with local differences between images [78]. These methods are capable of warping the target image locally to align it with the reference image. Non-rigid image registration is a challenging issue as a large number of parameters describing the spatial correspondence of images are needed. Most TPSs now support both rigid and deformable image registration and fusion [79].

The advances in technology have led to an escalation of the prescription dose or a change in the number of fractions. In recent years, increased attention has been paid to the radiobiological optimisation of the treatment plan, using TCP and NTCP models [14]. Even though dose-volume optimisation techniques are a mainstay of current TPSs, the biological optimisation used in IMRT planning is able to reduce radiation-induced toxicity [80-91].

In addition, although advanced inverse-planning techniques have led to the development of more automated approaches, plan optimisation is still a very time-consuming task with output varying greatly according to the experience of the operator. At the moment, the move towards using fully automated TPSs clinically is slow and debates are ongoing [84]. The arguments against automated treatment planning have concentrated on the examples of those treatments that are difficult to do automatically, such as bilateral post-implant chest wall irradiation.

\section{Tumour localisation in treatment planning}

As mentioned earlier, the more precise radiation delivery becomes, the more important it is to accurately identify the extension of both the tumour mass and also the normal tissue and critical organs involved in the neoplastic degeneration. This is essential in order to optimise irradiation geometry by delivering the radiation dose to the tumour itself while minimizing the dose delivered to surrounding tissue and organs at risk (OARs).

The integration of radiological/metabolic imaging, such as MRI and PET, with the CT scan simulation can provide useful information for accurately visualizing the tumour volume $[16,17]$. The integration of these images is made possible by image registration algorithms incorporated into the TPS. Technological developments mean that MRI has become increasingly useful in identifying and characterizing lesions within the prostate as well as detecting local disease recurrence following primary definitive prostate treatment. The inclusion of multiple MRI parameters is known as multiparametric MRI (mpMRI). Overlapping modalities in mpMRI corrects for deficiencies inherent in any individual sequence. Combining advanced techniques of functional MRI, including T2 weighted (T2W), dynamic contrast enhanced and diffusion weighted imaging (DWI), improves visualisation and the accurate detection of intra-prostatic lesions and differentiates between low and intermediate/high-grade disease [18, 44]. PET with different tracers has made it possible to obtain metabolic information and identify 
the most radio-resistant sub-volumes within the tumour [19, 20]. Adequate contouring is the fundamental pre-requisite for an effective and safe treatment plan. However, it is a process prone to errors and inter- and intra-operator variability [85, 86]. The delineation of tumour volumes is based on a complex process of data interpretation (clinical history, pathology and imaging). To improve the consistency of contouring among radiation oncologists, several working groups have provided consensus instructions and atlases [87-91]. However, it should be noted that no one guideline can be adopted as the perfect recipe for all patients. These protocols are often derived from 'expert opinion' and consensus as well as objective evidence, so it is not surprising that there can be variation between groups. Besides, the possibility of microscopic spread of the tumour beyond the anatomic borders defined by the atlases should be carefully considered by radiation oncologists, especially when high-precision techniques are used.

Generally, with the introduction of IMRT techniques into the clinical scenario, a large number of OARs is delineated to assess the low-dose bath. A recent development in RT is the use of automated atlas-based auto-segmentation algorithms to support contouring OARs [92, 93]. Several studies have demonstrated that inter-observer variability decreases when the automatic atlas-based segmented structures are used as a base and then modified [94-97]. Automatic or semi-automatic (needing manual revision) segmentation algorithms can speed up the delineation of OARs and they offer reliability and repeatability in delineating the structures.

\section{Tumour localisation in treatment delivery}

The increasingly conformal dose distributions that are possible with modern RT make it even more important that the patient's position is the same for the simulation and for the treatment. The sensitivity to treatment uncertainties due to organ motion and inaccurate patient positioning is even more worrying when high-precision techniques are combined with dose escalation and hypo-fractionation schemes.

\section{Image-guided radiotherapy}

Technological innovations have made possible the direct integration of imaging technology into the radiation treatment device to increase the precision and accuracy of radiation delivery by controlling the placement of the dose within the body [22, 98].

A broad range of image-guided radiotherapy (IGRT) modalities is now available and generally used. There are several methods for localizing the target for each treatment fraction: by localizing surrogates, including implanted fiducial markers, external surface markers or anatomical features (through planar imaging, fluoroscopy, kilovoltage CT (kV-CT) or megavoltage CT (MV-CT), MRI, ultrasound and x-ray imaging, electromagnetic localisation, optical surface imaging and so on. Depending on the imaging methods used, the IGRT systems may broadly be divided into radiation based, non-radiation based and hybrid systems $[21,99,100]$.

Of all soft-tissue based IGRT techniques, cone beam CT (CBCT) is the most widely used. It consists of acquiring multiple projection radiographs (for head and neck imaging $\sim 350$, for thoracic/pelvic imaging up to 600 ) before the RT fraction and within a gantry rotation of $180^{\circ}-360^{\circ}$. A volumetric image with high spatial resolution and sufficient soft-tissue contrast is reconstructed and registered to the reference planning CT to determine the correct target position. Translational and rotational positioning errors can be corrected online before irradiation [23, 24, 101-103].

To mitigate the effects of tumour motion due to respiration on image quality and registration uncertainty, CBCT can be acquired in conjunction with breath-hold strategies [104] or in a respiratory triggered approach (4D-CBCT) [105]. Moreover, ultrafast 'snapshot' volume imaging is ready to be deployed clinically [106].

Surface imaging devices, either based on passive infrared markers or active markers, can be used as a complementary positioning device, providing intra-fraction patient surveillance which further improves the overall precision of the treatment [107-114].

IGRT has been improved by the development of devices making it possible to reposition the patient using MRI. MRI yields superb softtissue visualisation and provides several imaging modalities for identifying movement, function and physiology without delivering any additional dose to the patient. Various integrated MRI-guided radiation therapy systems have been developed, such as the hybrid MRI-LINAC $[115,116]$, which is still a prototype, and the Viewray system (MRIdian System, ViewRay, Inc., Cleveland Oakwood Village, OH, USA) [117], which has been in clinical use for the last couple of years. 
IGRT not only makes it possible to improve the accuracy of the treatment by minimizing the inter-fractional position uncertainties, but it is also able to monitor systematic changes in the shape and position of tumour volume and of normal tissue (weight loss and tumour regression), which means that the plan can be suitably modified.

\section{Breathing adaptive radiotherapy}

Real-time monitoring of patient position significantly reduces intra-fraction movement, due either to physiological movement as in the case of the prostate, or due to respiration when tumours are located in the lung or upper abdomen. Electromagnetic technologies such as implanted radiofrequency markers have been successfully used for the prostate [118, 119]. Marker-based real-time image guidance has been in clinical use in the CyberKnife systems for over a decade. For its use to become widespread, real-time IGRT will probably need markerless solutions. A variety of kilovolt-based and MV-based possibilities have been proposed [120]. Cine MRI, which is available with the new MRI-guided radiation therapy systems, is able to provide non-invasive target localisation during RT treatment [121, 122].

The American Association of Physicists in Medicine Task group 76 [123] recommends caution when using IMRT techniques in regions where respiratory motion may jeopardise treatment. At least two intra-fraction motion effects may be present in the intensity-modulated treatment of a target subject to respiratory-induced motion: the dose blurring and the interplay effect. Planning studies show that during IMRT treatments a cyclic breathing motion with a 5-30-mm amplitude has a significant effect on dosimetry [124-126].

Several techniques have been proposed to reduce the effects of respiratory motion, from simple motion-encompassing, respiratory gating, either in free-breathing or in breath hold, to dynamic tumour tracking (DTT) [116, 127]. With the motion-encompassing technique, the mean position and the range of tumour motion are estimated from fluoroscopy or four-dimensional CT (4D-CT) data to define the internal target volume (ITV). However, a large ITV is required to accommodate tumour motion, which may lead to an increase in normal tissue complications. Respiratory gating, in free-breathing or in breath-hold, reduces the size of the ITV, but it increases the overall treatment time since the irradiation is delivered only in a predefined phase of the respiratory cycle, usually end expiration or end inspiration (in the case of deep inspiration breath hold), and it might be uncomfortable for the patient [127]. However, deep inspiration breath hold reduces the dose to the heart and left lung in the case of irradiation to the left breast [128]. The most sophisticated and efficient technique to reduce the size of the ITV is DTT, which makes it possible to reposition the beam dynamically according to the position of the tumour once the real-time IGRT system has determined tumour location. This can be done by repositioning the radiation beam to the target using a dynamic MLC [128], a robotic LINAC (CyberKnife) [130] or a linac mounted on two orthogonal gimbals allowing pan and tilt rotation of the beam, VERO [113, 131]. Another method, which has been discussed in several studies but has yet to be clinically implemented, consists of repositioning the patient to the beam by means of a dynamic couch [132]. A recently published comparison of the four tracking technologies showed that all systems are capable of highly accurate target delivery in the presence of motion, and that large treatment errors resulted when motion was not explicitly accounted for [133].

\section{Adaptive radiotherapy}

ART and dose painting strategies are the most recent tailored treatments.

ART is a closed-loop radiation treatment process where the treatment plan can be adjusted using measurement feedback. The term ART usually refers to: 1) changing the treatment plan during a course of RT to account for temporal changes in anatomy (e.g. tumour shrinkage, weight loss, internal motion or change of OARs), 2) adjustment of the delivered dose based on early tumour response and 3) adaptation of the treatment strategy based on early response (e.g. adding chemotherapy or hypoxic sensitisers).

ART is very much dependent on the anatomical information provided by IGRT and relies on deformable image registration algorithms now incorporated in several TPSs [13]. Image-guidance cannot fully correct for non-rigid changes and, in general, a single plan produced before treatment is not sufficient to describe the actual delivered doses and often leads to suboptimal treatment. Treatment delivery can be changed daily or weekly to compensate for changes in patient anatomy (e.g. tumour shrinkage, weight loss or internal motion) as seen on the images acquired daily at the treatment unit. Various techniques have been put forward, from the simple off-line strategies based on a limited number of imaging data to the more sophisticated 'plan of the day' approach which makes it possible to compensate for day-by-day variations in the target volume and position. 
The distribution of the delivered dose can be adapted to changes in tumour biology/function (e.g. hypoxia) as measured by functional imaging acquired during the course of treatment [25].

This technique requires advanced TPSs that are able to perform deformable image registration to take into account the changes in the target volume and surrounding OARs and the dose accumulation algorithm. The latter makes it possible to calculate the dose distribution on each imaging set (CT or $\mathrm{CBCT}$ ) and then to calculate the delivered dose distribution as the accumulation of the single treatment plans.

A more appealing approach is the integration of molecular imaging into the anatomical information with the aim of identifying radiationresistant regions within the tumour, such as high clonogen density, proliferation or hypoxia, as different tumour regions have a different radiosensitivity, which may make a heterogeneous dose distribution desirable in order to obtain greater tumour control.

There are two methods of achieving dose painting to biologic image-defined regions within a target: dose painting by contours and dose painting by numbers $[134,135]$. The first prescribes the dose within biological image-defined contours of the target, while the second prescribes dose to voxels throughout the target as a function of signal intensity of the corresponding voxel in a biologic image. One critical issue is the ability to accurately visualise in space and time the exact location of those areas expressing a phenotype that may require the delivery of a higher radiation dose. This raises not only the availability of specific tracers for the various biologic pathways of interest but also the spatial resolution of the available imaging modalities. Furthermore, the dynamics of tumour biology with its temporal and spatial changes have to be taken into account.

The implementation of ART schemes presents several challenges. It requires the acquisition of repeated anatomical and/or functional images during treatment and the use of deformable image registration algorithms for target volume propagation and plan summation. It is still therefore rarely used in clinical practice because it is so time-consuming.

\section{Biological advances in tumour targeting}

RT is moving towards the era of personalised medicine and away from the wrong assumption that 'one size fits all' and is offering individualised treatment strategies. The focus on individual variability will lead to a paradigm shift from common population based medicine to personalised and participative medicine [136, 137].

As we know, the efficacy of RT is limited by the intrinsic radioresistance of tumour cells, which means an increased risk of local tumour recurrence, so the need to overcome radioresistance and improve radiosensitivity explains why there is such great interest in identifying new molecules that have a synergistic effect with radiation.

One way to enhance the efficacy of RT that is already in use is to give chemotherapy or targeted agents concomitantly in order to modify the radiosensitivity of the tumour cells at the molecular level [138]. This field of radiation and cancer biology is rapidly expanding to provide a selective improvement in the tumour response to radiation, including T-cell checkpoint inhibitors, hypoxic radiosensitisers and cytotoxins, antiangiogenic agents, DNA repair inhibitors, signal transduction blockers, chemokine inhibitors and oxygen metabolism modifiers. Thus, there is a huge gap between the many exciting ideas emerging from pre-clinical studies in modern radiation and tumour biology and the lack of clinical trials testing these new concepts [139].

Furthermore, the immunotherapy field offers exciting prospects, with a drastic increase in immunotherapeutic protocols within the standard anticancer regimens. Similar progress is being made in radiobiological knowledge, as reported in a recently published review on the relation between RT and immunotherapy. What appears clear is that: 1) RT not only has a direct cytotoxic effect on tumour cells, but also reprograms the tumour microenvironment to exert a potent antitumour immune response, 2) RT enhances antitumour immunity, but also induces immunosuppressive responses and 3) the combination of immunotherapy and RT presents a multimodal treatment approach that involves stimulating and suppressing various pathways [140].

While numerous pre-clinical studies on RT-immunotherapy combination regimens have been reported, it is obviously necessary to carry out clinical and translational studies to explore what are the optimal RT doses, fractionation, timing and sequencing, and how they interact with various kinds of immunotherapy. Moreover, therapeutic success depends on the careful selection of immunotherapy agents and suitable patients [141]. 
At the same time, attempts are being made to identify the factors which might help to predict patients' response to RT treatment. Predicting the response to RT by distinguishing between radioresistant and radiosensitive patients could be useful in minimising the risk of unnecessary treatment and the related side effects. Identifying biomarkers that can predict the sensitivity or resistance of tumours to radiation therapy and the risk of developing toxicity is another promising area of research [142].

Several investigators are attempting to apply the field of 'omics' to tailor individual treatment in order to obtain a better outcome in cancer therapy by means of the expression of genes, proteins and metabolites [143]. In radiation oncology, 'omics' may be able to predict the treatment response by screening for genetic polymorphism or by gene expression analysis, and assessing the potential of epigenetic factors, posttranslational modification, signal transduction and metabolism. An example in the plethora of 'omics studies' was published recently: a patient-specific molecular signature of radiation sensitivity used to identify the optimum RT dose; a gene expression-based radiationsensitivity index and the linear quadratic model to derive the genomic-adjusted radiation dose (GARD) [144].

Besides the above mentioned 'radiogenomics', another promising area of ongoing research is 'radiomics' which aims to identify noninvasive imaging biomarkers. Radiomics analyses numerous medical imaging features, to which are added critical and interchangeable information regarding tumour phenotype [145]. In addition, radiomic features provide a complete and full representation of the entire tumour and capture intra-tumour heterogeneity. Radiomic features have been shown to be related to tumour histology, tumour grade/stage, patient survival, metabolism and other clinical features. Furthermore, some radiogenomic studies have reported an association between radiomic features and the underlying gene expression patterns [146].

It is thought that intra-tumour heterogeneity has potentially profound implications for clinical prediction (i.e. treatment response, survival outcomes, disease progression, etc) and consequently it is deemed to be an essential element of precision oncology.

\section{Conclusion}

In conclusion, RT has undergone tremendous progress over the years, realising technological developments that have revolutionised its clinical use, but we must not forget the multifaceted nature of this discipline that makes it an interface between physics, chemistry, biology and medicine [147]. Only by exploring all these aspects will we manage to produce individualised radiation therapy with better target delineation, avoidance of normal tissue, dose escalation, dose fractionation and better prediction of treatment response.

\section{Acknowledgments}

This study was partly supported by the Associazione Italiana per la Ricerca sul Cancro, project IG-13218 'Short-term high precision RT for early PC with concomitant boost to the dominant lesion', registered at ClinicalTrials.gov (NCT01913717), approved by the Instituto Europeo di Oncologia S768/113; by a research grant from Accuray Inc. titled 'Data collection and analysis of Tomotherapy and CyberKnife breast clinical studies, breast physics studies and prostate study' and by the Fondazione Umberto Veronesi project title 'AXILL-ART: Biology-based RT volume definition for 1-2 macrometastatic sentinel lymph nodes without further dissection in breast cancer conservative surgery'. The authors would like to thank Mrs. Verlie Anne Jones, dosimetrist in our department, for her valuable contribution towards editing the paper.

\section{Author contributions}

Cristina Garibaldi and Barbara Alicja Jereczek-Fossa equally contributed to the work and should be considered as first authors. 


\section{References}

1. Barton MB, Frommer M, and Shafiq J (2006) Role of radiotherapy in cancer control in low-income and middle-income countries Lancet Oncol 7 584-595 https://doi.org/10.1016/S1470-2045(06)70759-8 PMID: 16814210

2. Barton MB, Jacob S, and Shafiq J, et al (2014) Estimating the demand for radiotherapy from the evidence: a review of changes from 2003 to 2012 Radiother Oncol 112 140-144 https://doi.org/10.1016/j.radonc.2014.03.024 PMID: 24833561

3. Tyldesley S, Delaney G, and Foroudi F, et al (2011) Estimating the need for radiotherapy for patients with prostate, breast, and lung cancers: verification of model estimates of need with radiotherapy utilization data from British Columbia Int $J$ Radiat Oncol Biol Phys 79 1507-1515 https://doi.org/10.1016/j.jirobp.2009.12.070

4. Alterio D, Franco P, and Numico G, et al (2016) Non-surgical organ preservation strategies for locally advanced laryngeal tumors: What is the Italian attitude? Results of a national survey on behalf of AIRO and AIOM Med Oncol 3376 https://doi. org/10.1007/s12032-016-0781-5 PMID: 27290695

5. Gerardi MA, Jereczek-Fossa BA, and Zerini D, et al (2016) Bladder preservation in non-metastatic muscle-invasive bladder cancer (MIBC): a single-institution experience Ecancermedicalscience 10657 https://doi.org/10.3332/ecancer.2016.657 PMID: 27563352 PMCID: $\underline{4970626}$

6. Mak RH, Zietman AL, and Heney NM, et al (2008) Bladder preservation: optimizing radiotherapy and integrated treatment strategies BJU Int 102 1345-1353 https://doi.org/10.1111/j.1464-410X.2008.07981.x PMID: 19035903

7. Fisher B, Anderson S, and Redmond CK, et al (1995) Reanalysis and results after 12 years of follow-up in a randomized clinical trial comparing total mastectomy with lumpectomy with or without irradiation in the treatment of breast cancer $N$ Engl $\mathrm{J}$ Med 333 1456-1461 https://doi.org/10.1056/NEJM199511303332203 PMID: 7477145

8. Salvador-Coloma C and Cohen E (2016) Multidisciplinary care of laryngeal cancer J Oncol Pract 12 717-724 https://doi.org/10.1200/ JOP.2016.014225 PMID: $\underline{27511718}$

9. Chow E, Zeng L, and Salvo N, et al (2012) Update on the systematic review of palliative radiotherapy trials for bone metastases Clin Oncol (R Coll Radiol) 24 112-124 https://doi.org/10.1016/j.clon.2011.11.004

10. Hellman S (1997) Stopping metastases at their source N Engl J Med 337 996-997 https://doi.org/10.1056/NEJM199710023371408 PMID: 9309106

11. Golden EB, Chhabra A, and Chachoua A, et al (2015) Local radiotherapy and granulocyte-macrophage colony-stimulating factor to generate abscopal responses in patients with metastatic solid tumours: a proof-of-principle trial Lancet Oncol 16 795-803 https://doi.org/10.1016/S1470-2045(15)00054-6 PMID: 26095785

12. Budach W, Bölke E, and Matuschek C (2015) Hypofractionated radiotherapy as adjuvant treatment in early breast cancer: a review and meta-analysis of randomized controlled trials Breast Care (Basel) 10 240-245 https://doi.org/10.1159/000439007

13. Brock KK, Mutic S, and McNutt TR, et al (2017) Use of image registration and fusion algorithms and techniques in radiotherapy: Report of the AAPM Radiation Therapy Committee Task Group No. 132 Med Phys https://doi.org/10.1002/mp.12256

14. Nahum AE and Uzan J (2012) (Radio) biological optimization of external-beam radiotherapy Comput Math Methods Med 329214 https://doi.org/10.1155/2012/329214

15. Metcalfe P, Liney GP, and Holloway L, et al (2013) The potential for an enhanced role for MRI in radiation-therapy treatment planning Technol Cancer Res Treat 12 429-446 https://doi.org/10.7785/tcrt.2012.500342 PMID: 23617289 PMCID: 4527434 
16. Giezen M, Kouwenhoven E, and Scholten AN, et al (2011) Magnetic resonance imaging - versus computed tomography-based - target volume delineation of the glandular breast tissue (clinical target volume breast) in breast-conserving therapy: an exploratory study Int J Radiat Oncol Biol Phys 81 804-811 https://doi.org/10.1016/j.jijrobp.2010.07.004

17. Cho O, Chun M, and Oh YT, et al (2013) Can initial diagnostic PET-CT aid to localize tumor bed in breast cancer radiotherapy: feasibility study using deformable image registration Radiat Oncol 8163 https://doi.org/10.1186/1748-717X-8-163 PMID: 23822720 PMCID: $\underline{3720271}$

18. Dulaney CR, Osula DO, and Yang ES, et al (2016) Prostate radiotherapy in the era of advanced imaging and precision medicine Prostate Cancer 4897515 https://doi.org/10.1155/2016/4897515 PMID: 27022486 PMCID: 4771898

19. Ashamalla H, Rafla S, and Parikh K, et al (2005) The contribution of integrated PET/CT to the evolving definition of treatment volumes in radiation treatment planning in lung cancer Int J Radiat Oncol Biol Phys 63 1016-1023 https://doi.org/10.1016/j. ijrobp.2005.04.021 PMID: 15979817

20. De Ruysscher D, Nestle U, and Jeraj R, et al (2012) PET scans in radiotherapy planning of lung cancer Lung Cancer 75 141-145 https://doi.org/10.1016/j.lungcan.2011.07.018

21. Franzone P, Fiorentino A, and Barra S, et al (2016) Image-guided radiation therapy (IGRT): practical recommendations of Italian Association of Radiation Oncology (AIRO) Radiol Med 121 958-965 https://doi.org/10.1007/s11547-016-0674-x PMID: 27601141

22. Jaffray DA (2012) Image-guided radiotherapy: from current concept to future perspectives Nat Rev Clin Onco/ 9 688-699 https:// doi.org/10.1038/nrclinonc.2012.194 PMID: 23165124

23. Nabavizadeh N, Elliott DA, and Chen Y, et al (2016) Image guided radiation therapy (IGRT) practice patterns and IGRT's impact on workflow and treatment planning: results from a national survey of American Society for Radiation Oncology members Int J Radiat Oncol Biol Phys 94 850-857 https://doi.org/10.1016/j.jirobp.2015.09.035 PMID: 26972658

24. Ariyaratne $\mathrm{H}$, Chesham $\mathrm{H}$, and Alonzi $\mathrm{R}$ (2017) Image-guided radiotherapy for prostate cancer in the United Kingdom: a national survey $\mathrm{Br} J$ Radiol 9020160059 https://doi.org/10.1259/bir.20160059

25. Grau C, Muren LP, and Høyer M, et al (2008) Image-guided adaptive radiotherapy-integration of biology and technology to improve clinical outcome Acta Oncol 47 1182-1185 https://doi.org/10.1080/02841860802282802

26. Mothersill C and Seymour C (2001) Radiation-induced bystander effects: past history and future directions Radiat Res 155 759-767 https://doi.org/10.1667/0033-7587(2001)155[0759:RIBEPH]2.0.CO;2 PMID: 11352757

27. Turesson I, Carlsson J, and Brahme A, et al (2003) Biological response to radiation therapy Acta Oncol 42 92-106 https://doi. org/10.1080/02841860310004959 PMID: 12801128

28. Huang SM, Bock JM, and Harari PM (1999) Epidermal growth factor receptor blockade with C225 modulates proliferation, apoptosis, and radiosensitivity in squamous cell carcinomas of the head and neck Cancer Res 59 1935-1940 PMID: 10213503

29. Spalding AC and Lawrence TS (2006) New and emerging radiosensitizers and radioprotectors Cancer Invest 24 444-456 https:// doi.org/10.1080/07357900600705706 PMID: 16777698

30. Shewach DS and Lawrence TS (2007) Antimetabolite radiosensitizers J Clin Oncol $254043-4050$ https://doi.org/10.1200/ JCO.2007.11.5287 PMID: $\underline{17827452}$

31. Jackson SP and Bartek J (2009) The DNA-damage response in human biology and disease Nature 461 1071-1078 https://doi. org/10.1038/nature08467 PMID: 19847258 PMCID: $\underline{2906700}$

32. Sheehan JP, Shaffrey ME, and Gupta B, et al (2010) Improving the radiosensitivity of radioresistant and hypoxic glioblastoma Future Oncol 6 1591-1601 https://doi.org/10.2217/fon.10.123 PMID: 21062158 
33. Khan FM (2009) The Physics of Radiation Therapy 4th edn (Philadelphia: Lippincott Williams \& Wilkins) pp 6-7

34. Baumann M and Petersen C (2005) TCP and NTCP: a basic introduction Rays 30 99-104 PMID: 16294901

35. Hubenak JR, Zhang Q, and Branch CD, et al (2014) Mechanisms of injury to normal tissue after radiotherapy: a review Plast Reconstr Surg 133 49e-56e https://doi.org/10.1097/01.prs.0000440818.23647.0b PMID: 24374687 PMCID: 3921068

36. Begg AC, Stewart FA, and Vens C, et al (2011) Strategies to improve radio-therapy with targeted drugs Nat Rev Cancer 11 239-253 https://doi.org/10.1038/nrc3007 PMID: 21430696

37. Withers HR (1975) The four R's of radiotherapy Advances in Radiation Biology eds In: Lett JT Lett, and Adler H Adler, editors. (New York: Academic Press) pp. 241-271 https://doi.org/10.1016/B978-0-12-035405-4.50012-8

38. Steel GG, McMillan TJ, and Peacock JH (1989) The 5 Rs of radiobiology Int J Radiat Biol $\mathbf{5 6} 1045-1048$ https://doi. org/10.1080/09553008914552491 PMID: 2574214

39. Miralbell R, Roberts SA, and Zubizarreta E, et al (2012) Dose-fractionation sensitivity of prostate cancer deduced from radiotherapy outcomes of 5,969 patients in seven international institutional datasets: $\mathbf{a} / \mathbf{b}=1.4$ (0.9-2.2) Gy Int J Radiat Oncol Biol Phys 82 17-24 https://doi.org/10.1016/j.jirobp.2010.10.075

40. Brenner DJ and Hall EJ (1999) Fractionation and protraction for radiotherapy of prostate carcinoma Int J Radiat Oncol Biol Phys 43 1095-1101 https://doi.org/10.1016/S0360-3016(98)00438-6 PMID: 10192361

41. Whelan TJ, Kim DH, and Sussman J (2008) Clinical experience using hypofractionated radiation schedules in breast cancer Semin Radiat Oncol 18 257-264 https://doi.org/10.1016/j.semradonc.2008.04.008 PMID: 18725113

42. Owen JR, Ashton A, and Bliss JM, et al (2006) Effect of radiotherapy fraction size on tumour control in patients with earlystage breast cancer after local tumour excision: long-term results of a randomised trial Lancet Oncol $7467-471$ https://doi. org/10.1016/S1470-2045(06)70699-4 PMID: 16750496

43. Ray KJ, Sibson NR, and Kiltie AE (2015) Treatment of breast and prostate cancer by hypofractionated radiotherapy: potential risks and benefits Clin Oncol (R Coll Radiol) 27 420-426 https://doi.org/10.1016/j.clon.2015.02.008

44. De Bari B, Arcangeli S, and Ciardo D, et al (2016) Extreme hypofractionation for early prostate cancer: biology meets technology Cancer Treat Rev 50 48-60 https://doi.org/10.1016/j.ctrv.2016.08.005 PMID: 27631875

45. Veldeman L, Madani I, and Hulstaert F, et al (2008) Evidence behind use of intensity-modulated radiotherapy: a systematic review of comparative clinical studies Lancet Oncol 9 367-375 https://doi.org/10.1016/S1470-2045(08)70098-6 PMID: 18374290

46. Jin JY, Wen N, and Ren L, et al (2011) Advances in treatment techniques: arc-based and other intensity modulated therapies Cancer J 17 166-176 https://doi.org/10.1097/PPO.0b013e31821f8318 PMID: 21610470

47. Mackie TR, Holmes T, and Swerdloff S, et al (1993) Tomotherapy: a new concept for the delivery of dynamic conformal radiotherapy Med Phys 20 1709-1719 https://doi.org/10.1118/1.596958 PMID: 8309444

48. Otto K (2008) Volumetric modulated arc therapy: IMRT in a single gantry arc Med Phys 35310-317 https://doi.org/10.1118/1.2818738 PMID: 18293586

49. Hamilton DG, Bale R, and Jones C, et al (2016) Impact of tumour bed boost integration on acute and late toxicity in patients with breast cancer: a systematic review Breast 27 126-135 https://doi.org/10.1016/j.breast.2016.03.002 PMID: 27113229

50. Nitsche M, Dunst J, and Carl UM, et al (2015) Emerging role of hypofractionated radiotherapy with simultaneous integrated boost in modern radiotherapy of breast cancer Breast Care (Basel) 10 320-324 https://doi.org/10.1159/000436951 
51. Franceschini D, Paiar F, and Meattini I, et al (2013) Simultaneous integrated boost-intensity-modulated radiotherapy in head and neck cancer Laryngoscope 123 E97-E103 https://doi.org/10.1002/lary.24257 PMID: 23775348

52. Orlandi E, Palazzi M, and Pignoli E, et al (2010) Radiobiological basis and clinical results of the simultaneous integrated boost (SIB) in intensity modulated radiotherapy (IMRT) for head and neck cancer: a review Crit Rev Oncol Hematol 73 11-25 https:// doi.org/10.1016/j.critrevonc.2009.03.003

53. Timon G, Ciardo D, and Bazani A, et al (2016) Rationale and protocol of AIRC IG-13218, short-term radiotherapy for early prostate cancer with concomitant boost to the dominant lesion Tumori 102 536-540 https://doi.org/10.5301/tj.5000547 PMID: $\underline{27514314}$

54. Fonteyne V, Villeirs G, and Speleers B, et al (2008) Intensity-modulated radiotherapy as primary therapy for prostate cancer: report on acute toxicity after dose escalation with simultaneous integrated boost to intraprostatic lesion Int $J$ Radiat Oncol Biol Phys 72 799-807 https://doi.org/10.1016/j.jijrobp.2008.01.040 PMID: 18407430

55. Hall EJ and Wuu CS (2003) Radiation-induced second cancers: the impact of 3D-CRT and IMRT Int J Radiat Oncol Biol Phys 56 83-88 https://doi.org/10.1016/S0360-3016(03)00073-7 PMID: 12694826

56. Berrington de Gonzalez A, Curtis RE, and Kry SF, et al (2011) Proportion of second cancers attributable to radiotherapy treatment in adults: a cohort study in the US SEER cancer registries Lancet Oncol 12 353-360 https://doi.org/10.1016/S14702045(11)70061-4 PMID: 21454129 PMCID: $\underline{3086738}$

57. Potters L, Kavanagh B, and Galvin J (2010) American Society for Therapeutic Radiology and Oncology (ASTRO) and American College of Radiology (ACR) practice guideline for the performance of stereotactic body radiation therapy Int $J$ Radiat Oncol Biol Phys 76 326-332 https://doi.org/10.1016/j.jijrobp.2009.09.042 PMID: 20117285

58. Aznar M, Romero AM, and Heijmen BJM (2017) The role of technology in clinical trials using stereotactic body radiotherapy $\mathrm{Br}$ J Radiol 9020160930 https://doi.org/10.1259/bjr.20160930 PMID: 28055252 PMCID: $\underline{601528}$

59. Narayanasamy G, Saenz D, and Cruz W, et al (2016) Commissioning an Elekta Versa HD linear accelerator J Appl Clin Med Phys 17 179-191 https://doi.org/10.1120/jacmp.v17i1.5799 PMID: 26894351

60. Glide-Hurst C, Bellon M, and Foster R, et al (2013) Commissioning of the Varian TrueBeam linear accelerator: a multi-institutional study Med Phys 40031719 https://doi.org/10.1118/1.4790563

61. Adler Jr JR, Chang SD, and Murphy MJ, et al (1997) The Cyberknife: a frameless robotic system for radiosurgery Stereotact Funct Neurosurg 69 124-128 https://doi.org/10.1159/000099863 PMID: 9711744

62. Solberg TD, Medin PM, and Ramirez E, et al (2014) Commissioning and initial stereotactic ablative radiotherapy experience with Vero J Appl Clin Med Phys 154685 https://doi.org/10.1120/jacmp.v15i2.4685 PMID: 24710458

63. Orecchia R, Surgo A, and Muto M, et al (2016) VERO 8 radiotherapy for low burden cancer: 789 patients with 957 lesions Ecancermedicalscience 10677 https://doi.org/10.3332/ecancer.2016.677

64. Abbas G, Danish A, and Krasna MJ (2016) Stereotactic body radiotherapy and ablative therapies for lung cancer Surg Oncol Clin N Am 25 553-566 https://doi.org/10.1016/j.soc.2016.02.008 PMID: 27261915

65. Durante M, Orecchia R, and Loeffler JS (2017) Charged-particle therapy in cancer: clinical uses and future perspectives Nat Rev Clin Oncol 14(8) 483-495 doi: 0.1038/nrclinonc.2017.30. Epub 2017 Mar 14. Review https://doi.org/10.1038/nrclinonc.2017.30 PMID: $\underline{28290489}$

66. Mohan R and Grosshans D (2017) Proton therapy-present and future Adv Drug Deliv Rev $10926-44$ https://doi.org/10.1016/j. addr.2016.11.006 
67. Fossati P, Vavassori A, and Deantonio L, et al (2016) Review of photon and proton radiotherapy for skull base tumours Rep Pract Oncol Radiother 21 336-355 https://doi.org/10.1016/j.rpor.2016.03.007 PMID: 27330419 PMCID: 4899429

68. Kubiak T (2016) Particle therapy of moving targets-the strategies for tumour motion monitoring and moving targets irradiation Br J Radio/ 8920150275 https://doi.org/10.1259/bjr.20150275

69. Bert C and Durante M (2011) Motion in radiotherapy: particle therapy Phys Med Biol 56 R113-R144 https://doi.org/10.1088/00319155/56/16/R01 PMID: 21775795

70. Ciocca M, Mirandola A, and Molinelli S, et al (2016) Commissioning of the 4-D treatment delivery system for organ motion management in synchrotron-based scanning ion beams Phys Med 32 1667-1671 https://doi.org/10.1016/j.ejmp.2016.11.107 PMID: $\underline{27890567}$

71. Matloob SA, Nasir HA and Choi D (2016) Proton beam therapy in the management of skull base chordomas: systematic review of indications, outcomes, and implications for neurosurgeons. Br J Neurosurg 30, 382-7 https://doi.org/10.1080/02688697.2016 $\underline{.1181154}$ PMID: $\underline{27173123}$

72. Mishra K and Daftari IK (2016) Proton therapy for the management of uveal melanoma and other ocular tumors Chin Clin Oncol 550 https://doi.org/10.21037/cco.2016.07.06 PMID: 27558251

73. Leroy R, Benahmed N, and Hulstaert F, et al (2016) Proton therapy in children: a systematic review of clinical effectiveness in 15 pediatric cancers Int J Radiat Oncol Biol Phys 95 267-278 https://doi.org/10.1016/.ijrobp.2015.10.025 PMID: 27084646

74. Ebner DK and Kamara T (2016) The emerging role of carbon-ion radiotherapy Front Oncol 7(6) 140 https://doi.org/10.3389/ fonc.2016.00140.

75. Kamada T, Tsuji H, and Blakely EA, et al (2015) Carbon ion radiotherapy in Japan: an assessment of 20 years of clinical experience Lancet Oncol 16(2) e93-e100 doi: 10.1016/S1470-2045(14)70412-7 https://doi.org/10.1016/S1470-2045(14)70412-7 PMID: $\underline{25638685}$

76. Matsufuji N (2017) Overview summary of clinical heavier-ion progress in Japan J Phys Conf Ser 860(1) art. no. 012027 https:// doi.org/10.1088/1742-6596/860/1/012027

77. Rossi S (2015) The National Centre for Oncological Hadrontherapy (CNAO): status and perspectives Phys Med 31 333-351 https://doi.org/10.1016/j.ejmp.2015.03.001 PMID: 25840619

78. Crum WR, Hartkens T, and Hill DL (2004) Non-rigid image registration: theory and practice Br J Radiol 77 140-153 https://doi. org/10.1259/bjr/25329214

79. Keszei AP, Berkels B, and Deserno TM (2017) Survey of non-rigid registration tools in medicine J Digit Imaging 30 102-116 https:// doi.org/10.1007/s10278-016-9915-8

80. Qi XS, Semenenko VA, and Li XA (2009) Improved critical structure sparing with biologically based IMRT optimization Med Phys 36 1790-1799 https://doi.org/10.1118/1.3116775 PMID: 19544798

81. Das S (2009) A role for biological optimization within the current treatment planning paradigm Med Phys $364672-4682$ https:// doi.org/10.1118/1.3220211 PMID: $\underline{19928099}$

82. Doit Q, Kavanagh B, and Timmerman R, et al (2012) Biological-based optimization and volumetric modulated arc therapy delivery for stereotactic body radiation therapy Med Phys 39 237-245 https://doi.org/10.1118/1.3668059

83. Liang X, Penagaricano J, and Zheng D, et al (2016) Radiobiological impact of dose calculation algorithms on biologically optimized IMRT lung stereotactic body radiation therapy plans Radiat Oncol 1110 https://doi.org/10.1186/s13014-015-0578-2 PMID: $\underline{26800883}$ PMCID: $\underline{4724090}$ 
84. Sharpe MB, Moore KL, and Orton CG (2014) Within the next ten years treatment planning will become fully automated without the need for human intervention Med Phys 41120601 https://doi.org/10.1118/1.4894496

85. Vinod SK, Min M, and Jameson MG, et al (2016) A review of interventions to reduce inter-observer variability in volume delineation in radiation oncology J Med Imaging Radiat Oncol 60 393-406 https://doi.org/10.1111/1754-9485.12462 PMID: 27170216

86. Vinod SK, Jameson MG, and Min M, et al (2016) Uncertainties in volume delineation in radiation oncology: a systematic review and recommendations for future studies Radiother Oncol 121 169-179 https://doi.org/10.1016/j.radonc.2016.09.009 PMID: $\underline{27729166}$

87. White J, Tai A, and Arthur D, et al (2016) Breast cancer atlas for radiation therapy planning: consensus definitions. Available at: www.rtog.org/

88. Offersen BV, Boersma LJ, and Kirkove C, et al (2015) ESTRO consensus guideline on target volume delineation for elective radiation therapy of early stage breast cancer Radiother Oncol 114 3-10 https://doi.org/10.1016/j.radonc.2014.11.030 PMID: 25630428

89. Nielsen MH, Berg M, and Pedersen AN, et al (2013) Delineation of target volumes and organs at risk in adjuvant radiotherapy of early breast cancer: national guidelines and contouring atlas by the Danish Breast Cancer Cooperative Group Acta Oncol $\mathbf{5 2}$ 703-710 https://doi.org/10.3109/0284186X.2013.765064 PMID: $\underline{23421926}$

90. Gentile MS, Usman AA, and Neuschler El, et al (2015) Contouring guidelines for the axillary lymph nodes for the delivery of radiation therapy in breast cancer: evaluation of the RTOG breast cancer atlas Int J Radiat Oncol Biol Phys 93 257-265 https:// doi.org/10.1016/j.ijrobp.2015.07.002 PMID: 26383674

91. Michalski JM, Lawton CA, and El-Naqa I, et al (2010) Development of RTOG consensus guidelines for the definition of the clinical target volume for postoperative conformal radiation therapy for prostate cancer Int J Radiat Oncol Biol Phys 76 361-368 https://doi.org/10.1016/j.jijrobp.2009.02.006 PMCID: $\underline{2847420}$

92. Iglesias JE and Sabuncu MR (2015) Multi-atlas segmentation of biomedical images: a survey Med Image Anal 24 205-219 https:// doi.org/10.1016/i.media.2015.06.012 PMID: 26201875 PMCID: 4 532640

93. Lim JY and Leech M (2016) Use of auto-segmentation in the delineation of target volumes and organs at risk in head and neck Acta Oncol 55 799-806 https://doi.org/10.3109/0284186X.2016.1173723 PMID: 27248772

94. Reed VK, Woodward WA, and Zhang L, et al (2009) Automatic segmentation of whole breast using atlas approach and deformable image registration Int J Radiat Oncol Biol Phys 73 1493-1500 https://doi.org/10.1016/j.j.jrobp.2008.07.001

95. Daisne JF and Blumhofer A (2013) Atlas-based automatic segmentation of head and neck organs at risk and nodal target volumes: a clinical validation Radiat Oncol 8154 https://doi.org/10.1186/1748-717X-8-154 PMID: 23803232 PMCID: $\underline{3722083}$

96. Stapleford LJ, Lawson JD, and Perkins C, et al (2010) Evaluation of automatic atlas-based lymph node segmentation for head and neck cancer Int J Radiat Oncol Biol Phys 77 959-966 https://doi.org/10.1016/j.jijrobp.2009.09.023 PMID: 20231069

97. Ciardo D, Gerardi MA, and Vigorito S, et al (2017) Atlas-based segmentation in breast cancer radiotherapy: evaluation of specific and generic-purpose atlases Breast 32 44-52 https://doi.org/10.1016/j.breast.2016.12.010

98. Verellen D, De Ridder M, and Linthout N, et al (2007) Innovations in image-guided radiotherapy Nat Rev Cancer $7949-960$ https:// doi.org/10.1038/nrc2288 PMID: $\underline{18034185}$

99. Bissonnette JP, Balter PA, and Dong L, et al (2012) Quality assurance for image-guided radiation therapy utilizing CT-based technologies: a report of the AAPM TG-179 Med Phys 39 1946-1963 https://doi.org/10.1118/1.3690466 PMID: 22482616

100. Willoughby T, Lehmann J, and Bencomo JA, et al (2012) Quality assurance for nonradiographic radiotherapy localization and positioning systems: report of Task Group 147 Med Phys 39 1728-1747 https://doi.org/10.1118/1.3681967 PMID: 22482598 
101. Garibaldi C, Piperno G, and Ferrari A, et al (2016) Translational and rotational localization errors in cone-beam CT based imageguided lung stereotactic radiotherapy Phys Med 32 859-865 https://doi.org/10.1016/j.ejmp.2016.05.055 PMID: 27289354

102. Garibaldi C, Russo S, and Ciardo D, et al (2015) Geometric and dosimetric accuracy and imaging dose of the real-time tumour tracking system of a gimbal mounted linac Phys Med 31 501-509 https://doi.org/10.1016/j.ejmp.2015.04.001 PMID: 25934523

103. Bian J, Sharp GC, and Park YK, et al (2016) Investigation of cone-beam CT image quality trade-off for image-guided radiation therapy Phys Med Biol 61 3317-3346 https://doi.org/10.1088/0031-9155/61/9/3317 PMID: 27032676

104. Josipovic M, Persson GF, and Bangsgaard JP, et al (2016) Deep inspiration breath-hold radiotherapy for lung cancer: impact on image quality and registration uncertainty in cone beam CT image guidance Br J Radiol 8920160544 https://doi.org/10.1259/ bjr.20160544 PMID: 27706950 PMCID: $\underline{5604920}$

105. O'Brien RT, Cooper BJ, and Shieh CC, et al (2016) The first implementation of respiratory triggered 4DCBCT on a linear accelerator Phys Med Biol 61 3488-3499 https://doi.org/10.1088/0031-9155/61/9/3488 PMID: 27051977

106. Blessing M, Stsepankou D, and Wertz H, et al (2010) Breath-hold target localization with simultaneous kilovoltage/megavoltage cone-beam computed tomography and fast reconstruction Int J Radiat Oncol Biol Phys 78 1219-1226 https://doi.org/10.1016/j. ïrobp.2010.01.030 PMID: 20554124

107. Moser T, Habl G, and Uhl M, et al (2013) Clinical evaluation of a laser surface scanning system in 120 patients for improving daily setup accuracy in fractionated radiation therapy Int J Radiat Oncol Biol Phys 85 846-853 https://doi.org/10.1016/j. iirobp.2012.05.026

108. Fassi A, Schaerer J, and Riboldi M, et al (2015) An image-based method to synchronize cone-beam CT and optical surface tracking J Appl Clin Med Phys 165152 https://doi.org/10.1120/jacmp.v16i2.5152 PMID: 26103183

109. Apicella G, Loi G, and Torrente S, et al (2016) Three-dimensional surface imaging for detection of intra-fraction setup variations during radiotherapy of pelvic tumors Radiol Med 121 805-810 https://doi.org/10.1007/s11547-016-0659-9 PMID: 27300649

110. Crop F, Pasquier D, and Baczkiewic A, et al (2016) Surface imaging, laser positioning or volumetric imaging for breast cancer with nodal involvement treated by helical TomoTherapy J App/ Clin Med Phys 17 1-12 https://doi.org/10.1120/jacmp.v17i5.6041

111. Ricotti R, Ciardo D, and Fattori G, et al (2017) Intra-fraction respiratory motion and baseline drift during breast Helical Tomotherapy Radiother Oncol 122 79-86 https://doi.org/10.1016/j.radonc.2016.07.019

112. Garibaldi C, Catalano G, and Baroni G, et al Deep inspiration breath-hold technique guided by an opto-electronic system for extracranial stereotactic treatments J Appl Clin Med Phys 144087

113. Garibaldi C, Jereczek-Fossa BA, and Zerini D, et al (2015) Image-guided radiotherapy for prostate cancer using 3 different techniques: localization data of 186 patients Tumori 101 273-280 https://doi.org/10.5301/ti.5000322 PMID: 25908027

114. Fontana G, Riboldi M, and Gianoli C, et al (2016) MRI quantification of pancreas motion as a function of patient setup for particle therapy - a preliminary study J Appl Clin Med Phys 17 1-16 https://doi.org/10.1120/jacmp.v17i5.6236

115. Lagendijk JJ, Raaymakers BW, and Raaijmakers AJ, et al (2008) MRI/linac integration Radiother Oncol 86 25-29 https://doi. org/10.1016/j.radonc.2007.10.034

116. Jia X, Tian Z, and Xi Y, et al (2017) New concept on an integrated interior magnetic resonance imaging and medical linear accelerator system for radiation therapy J Med Imaging (Bellingham) 4015004 https://doi.org/10.1117/1.JMI.4.1.015004

117. Yang Y, Cao M, and Sheng K, et al (2016) Longitudinal diffusion MRI for treatment response assessment: preliminary experience using an MRI-guided tri-cobalt 60 radiotherapy system Med Phys 43 1369-1373 https://doi.org/10.1118/1.4942381 PMID: $\underline{26936721}$ 
118. Giandini T, Panaino CM, and Avuzzi B, et al (2017) An accurate method to quantify breathing-induced prostate motion for patients implanted with electromagnetic transponders Tumori 103 136-142 https://doi.org/10.5301/tj.5000609 PMID: 28218381

119. Kupelian P, Willoughby T, and Mahadevan A, et al (2007) Multi-institutional clinical experience with the Calypso System in localization and continuous, real-time monitoring of the prostate gland during external radiotherapy Int J Radiat Oncol Biol Phys 67 1088-1098 https://doi.org/10.1016/j.jijrobp.2006.10.026

120. Richter A, Wilbert J, and Baier K, et al (2010) Feasibility study for markerless tracking of lung tumors in stereotactic body radiotherapy Int J Radiat Oncol Biol Phys 78 618-627 https://doi.org/10.1016/j.jirobp.2009.11.028 PMID: 20452143

121.Van Heijst TC, Philippens ME, and Charaghvandi RK, et al (2016) Quantification of intra-fraction motion in breast radiotherapy using supine magnetic resonance imaging Phys Med Biol 61 1352-1370 https://doi.org/10.1088/0031-9155/61/3/1352 PMID: $\underline{26797074}$

122. Seregni M, Paganelli C, and Lee D, et al (2016) Motion prediction in MRI-guided radiotherapy based on interleaved orthogonal cine-MRI Phys Med Biol 61 872-887 https://doi.org/10.1088/0031-9155/61/2/872 PMID: 26740517

123. Keall PJ, Mageras GS, and Balter JM, et al (2006) The management of respiratory motion in radiation oncology: report of AAPM Radiation Therapy Committee Task Group No. 76 Med Phys 33 3874-3900 https://doi.org/10.1118/1.2349696 PMID: 17089851

124. Kanagaki B, Read PW, and Molloy JA, et al (2007) A motion phantom study on helical tomotherapy: The dosimetric impacts of delivery technique and motion Phys Med Biol 52 243-255 https://doi.org/10.1088/0031-9155/52/1/016

125. Kissick MW, Boswell SA, and Jeraj R, et al (2005) Confirmation, refinement, and extension of a study in intrafraction motion interplay with sliding jaw motion Med Phys 32 2346-2350 https://doi.org/10.1118/1.1935774 PMID: 16121591

126. Cao J, Roeske JC, and Chmura SJ, et al (2009) Calculation and prediction of the effect of respiratory motion on whole breast radiation therapy dose distributions Med Dosim 34 126-132 https://doi.org/10.1016/j.meddos.2008.07.002 PMID: 19410141

127. Verellen D, Depuydt T, and Gevaert T, et al (2010) Gating and tracking, 4D in thoracic tumors Cancer Radiother $14446-454$ https:// doi.org/10.1016/j.canrad.2010.06.002 PMID: 20673737

128. Boda-Heggemann J, Knopf AC, and Simeonova-Chergou A, et al (2016) Deep inspiration breath hold-based radiation therapy: a clinical review Int J Radiat Oncol Biol Phys 94 478-492 https://doi.org/10.1016/i.jirobp.2015.11.049 PMID: 26867877

129.Pepin EW, Wu H, and Shirato $\mathrm{H}$ (2013) Use of dMLC for implementation of dynamic respiratory-gated radiation therapy Med Phys 40101708 https://doi.org/10.1118/1.4820534 PMID: $\underline{24089898}$ PMCID: $\underline{3785535}$

130. Hoogeman M, Prévost JB, and Nuyttens J, et al (2009) Clinical accuracy of the respiratory tumor tracking system of the cyberknife: assessment by analysis of log files Int J Radiat Oncol Biol Phys 74 297-303 https://doi.org/10.1016/j.j.jrobp.2008.12.041 PMID: 19362249

131. Kamino Y, Takayama K, and Kokubo M, et al (2006) Development of four-dimensional image guided radiotherapy system with gimbaled x-ray head Int J Radiat Oncol Biol Phys 66 271-278 https://doi.org/10.1016/j.ijrobp.2006.04.044 PMID: 16820270

132. Souza W, Naqvi S, and Yu C (2005) Real-time intra-fraction-motion tracking using the treatment couch: a feasibility study Phys Med Biol 50 4021-4033 https://doi.org/10.1088/0031-9155/50/17/007 PMID: 16177527

133. Colvill E, Booth J, and Nill S, et al (2016) A dosimetric comparison of real-time adaptive and non-adaptive radiotherapy: a multiinstitutional study encompassing robotic, gimbaled, multileaf collimator and couch tracking Radiother Oncol 119 159-165 https://doi.org/10.1016/j.radonc.2016.03.006 PMID: 27016171 PMCID: 4854175

134. Jentsch C, Beuthien-Baumann B, and Troost EGC, et al (2015) Validation of functional imaging as a biomarker for radiation treatment response Br J Radiol 8820150014 https://doi.org/10.1259/bjr.20150014 PMID: 26083533 PMCID: 4628525 
135. Galvin JM and De Neve W (2007) Intensity modulating and other radiation therapy devices for dose painting $J$ Clin Oncol 25 924-930 https://doi.org/10.1200/JC0.2007.10.6716 PMID: 17350940

136. Lambin P, Zindler J, and Vanneste BG, et al (2016) Decision support systems for personalized and participative radiation oncology Adv Drug Deliv Rev 109 131-153 https://doi.org/10.1016/j.addr.2016.01.006 PMID: 26774327

137. Leonardi MC, Ricotti R, and Dicuonzo S, et al (2016) From technological advances to biological understanding: the main steps toward high-precision RT in breast cancer. Breast 29 213-222 https://doi.org/10.1016/j.breast.2016.07.010 PMID: 27542556

138. Torres-Roca JF (2012) A molecular assay of tumor radiosensitivity: a roadmap towards biology-based personalized radiation therapy Per Med 9 547-557 https://doi.org/10.2217/pme.12.55 PMID: 23105945 PMCID: 3480204

139.Brown JM and Adler JR Jr (2015) Is equipment development stifling innovation in radiation oncology? Int J Radiat Oncol Biol Phys 92 713-714 https://doi.org/10.1016/j.jijrobp.2015.03.005 PMID: 26104924

140. Weichselbaum RR, Liang H, and Deng L, et al (2017) Radiotherapy and immunotherapy: a beneficial liaison? Nat Rev Clin Oncol https://doi.org/10.1038/nrclinonc.2016.211 PMID: 28094262

141. Ishihara D, Pop L, and Takeshima T, et al (2017) Rationale and evidence to combine radiation therapy and immunotherapy for cancer treatment Cancer Immunol Immunother 66 281-298 https://doi.org/10.1007/s00262-016-1914-6

142. Herskind C, Talbot CJ, and Kerns SL, et al (2016) Radiogenomics: a systems biology approach to understanding genetic risk factors for radiotherapy toxicity? Cancer Lett 382 95-109 https://doi.org/10.1016/j.canlet.2016.02.035 PMID: 26944314 PMCID: $\underline{5016239}$

143. Chung $\mathrm{CH}$, Wong S, and Ang KK, et al (2007) Strategic plans to promote head and neck cancer translational research within the radiation therapy oncology group: a report from the translational research program Int $J$ Radiat Oncol Biol Phys 69(2 Suppl) 67-78 https://doi.org/10.1016/j.jijrobp.2007.04.090

144.Scott JG, Berglund A, and Schell MJ, et al (2017) A genome-based model for adjusting radiotherapy dose (GARD): a retrospective, cohort-based study Lancet Oncol 18 202-211 https://doi.org/10.1016/S1470-2045(16)30648-9

145. Aerts HJ, Velazquez ER, and Leijenaar RT, et al (2014) Decoding tumour phenotype by noninvasive imaging using a quantitative radiomics approach Nat Commun 54006 PMID: 24892406 PMCID: 4059926

146. Nicolasjilwan M, Hu Y, and Yan C, et al (2015) Addition of MR imaging features and genetic biomarkers 2048 strengthens glioblastoma survival prediction in TCGA patients J Neuroradiol 42 212-221 https://doi.org/10.1016/j.neurad.2014.02.006

147.Allen C, Her S, and Jaffray DA (2017) Radiotherapy for cancer: present and future Adv Drug Deliv Rev 109 1-2 https://doi. org/10.1016/j.addr.2017.01.004 PMID: 28189183 